\title{
OPTICAL RESOLUTION OF BE STAR ENVELOPES
}

\author{
F. VAKILI, D. MOURARD AND P. STEE \\ OCA-Fresnel-GI2T, 06460 Caussols, France
}

\begin{abstract}
Probing the atmospheres of Be stars by means of Optical Long Baseline Interferometry (OLBI) is a method still in its infancy. Published observational results in this field can be counted on one hand. In this paper we introduce the principles of OLBI as they apply to observations of $\mathrm{Be}$ stars and review results from existing long baseline interferometers. Regular operation of OLBI is most effective when carried out in conjunction with standard techniques such as photometry and spectroscopy. With the next generation of synthetic arrays combining sub-milliarcsecond resolution and a spectral resolution better than 10000, OLBI should be able to address the direct detection of pulsation and magnetic fields.
\end{abstract}

\section{Introduction}

Optical resolution of early-type stars was pioneered by Hanbury Brown and collaborators in the 1960s with the Narrabri Stellar Intensity Interferometer in Australia (Hanbury Brown et al. 1974a). In an intensive study of the spectroscopic binary $\gamma^{2}$ Vel (WC8+07), the Narrabri team measured the angular diameter of the C III-IV emission region surrounding the WolfRayet component, which they found to be 5 times larger than the central star (Hanbury Brown et al. 1970). In another survey which combined interferometry and polarimetry, Hanbury Brown and co-workers set an upper limit of 6.5 percent on the variation of the angular size of $\beta$ Ori in two orthogonal polarizations (Hanbury Brown et al. 1974b). From a simple model based on electron scattering in a hot corona, they were able to estimate an upper limit of $2 \times 10^{-5} M_{\odot} \mathrm{y}^{-1}$ on the mass loss of this blue supergiant. More appropriate models for comparing predicted interferometric measurements to actual data were used to interpret the observations of the $O$ star $\zeta$ Pup (Cassinelli \& Hoffman 1975). The possibility of resolving the envelope of $\mathrm{Be}$ stars in the Balmer lines had already been formulated by Hanbury Brown et al. (1974b), who concluded that such observations demanded a much more sensitive instrument. By the time their observing programme stopped, the Narrabri group had already paved the way for future studies of extended atmospheres in early-type stars.

The invention of speckle interferometry by Labeyrie in 1970, and his successful operation of a prototype optical amplitude interferometer with physically separated telescopes in 1974, opened new perspectives for measuring the geometry of Be star envelopes (Labeyrie 1978). Since Be stars are generally quite distant, it is not surprising that speckle observations have only set upper limits on the apparent diameters of their envelopes in the Balmer lines (Blazit et al. 1977). Interestingly enough, a significant number 
of spectroscopic binaries which include a B or Be component were resolved during systematic speckle observations (McAlister \& Hartkopf 1988). $\beta$ Cep and $o$ And are examples of such multiple objects resolved with the Palomar 5-m telescope (Bonneau et al. 1980, Labeyrie et al. 1974). Besides the detection of multiplicity, patrol observations of $\mathrm{B}$ or Be stars using true imaging techniques up to the diffraction limit of large telescopes, enable us to predict episodes of close contact between the components. Optical long baseline interferometers can be used to study the nature and evolution of accretion disks, or the disks formed by tidal interactions in close binaries, as these disks usually give rise to emission-lines in the visible region.

\section{Optical Long Baseline Interferometry and Be Stars}

\subsection{An Introduction to OLBI}

OLBI can be defined as the art of synthesizing a giant optical telescope, of say $100-\mathrm{m}$ diameter, from an array of much smaller apertures placed at comparable distances. In principle, the angular resolution of such an array attains the diffraction limit of a giant telescope, but the smaller lightcollecting surface lowers the limiting magnitude. The theory and implementation of OLBI is similar to that of radio interferometry. The main difference lies in the nature of the noise (which is mostly photon noise), the dramatic effect of atmospheric turbulence, and the absence of phase-tracking amplifiers at visible wavelengths. Besides the difficult task of coherently combining primary beams in the presence of mechanical instabilities, atmospheric turbulence drastically complicates the operation of optical interferometers. At present, only about five interferometers are operational. A comparable number of aperture synthesis projects should start operating in the coming decade. Further information is available in review papers in more specialized colloquia (see for instance Robertson \& Tango 1994).

An interferometer can be thought of as a spatial filter which samples the two-dimensional Fourier transform of the brightness distribution of the object of interest. Each sample is a complex quantity called the visibility which is a function of spatial frequency $(u, v)$. The spatial frequency is defined as the ratio of the baseline to the mean wavelength at which the interferometer is operated. When $N$ telescopes are operated together, $N(N-1) / 2$ such spatial frequencies are measured simultaneously. This is often referred to as the instantaneous $u v$-plane coverage. If $N$ is large enough, say $N \geq 20$ as in the case of the radio Very Large Array, a snapshot can almost be obtained from a straightforward Fourier transform of visibility data. If $N$ is small, the domain of spatial sampling can be extended by using earth-rotation synthesis. With good $u v$-coverage, true images can be reconstructed using numerical inversion techniques. The dynamic range and detail in the reconstructed image depends both on the signal-to-noise ratio of measured visibilities and 


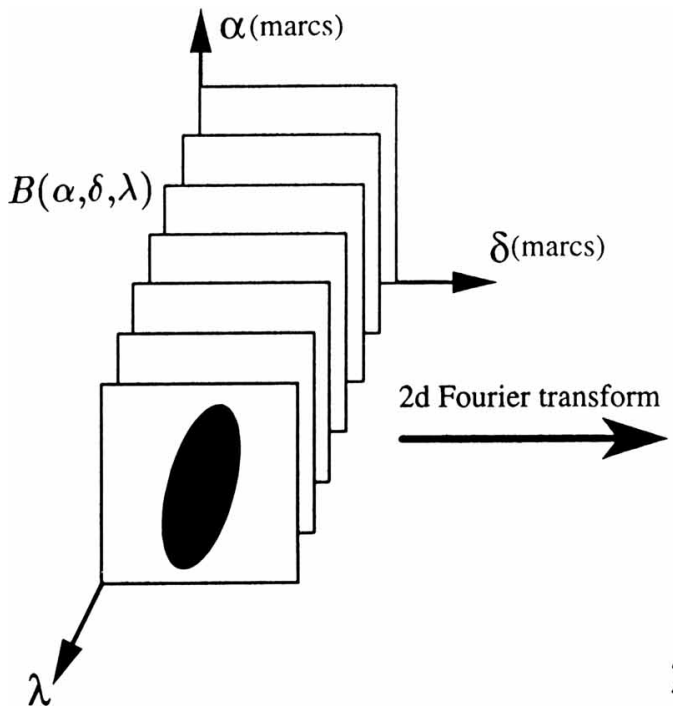

sky space:

angular coordinates, wavelength

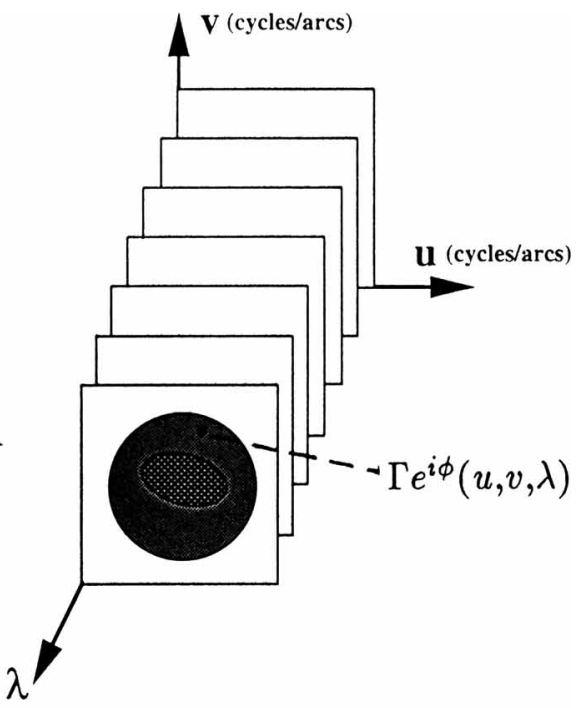

visibility space:

spatial frequency, wavelength

Fig. 1. Schematic representation of the brightness of a Be star as projected on the sky, $B(\alpha, \delta, \lambda)$, as a function of wavelength (left). For each $\lambda$-slice of $B$ there corresponds a complex visibility map $\Gamma e^{i \phi}(x, v, \lambda)$ which is the two-dimensional Fourier transform of $B$.

the $u v$-plane filling factor achieved during a synthesis run. Until now, only interferometers with two telescopes have produced data on Be stars.

\subsection{Application of OLBI to Be Stars}

Most Be stars are notoriously variable with a morphology that depends on the wavelength across various scales of angular and spectral resolutions. Therefore, if OLBI is to be used for deducing morphological structures, it would be most efficiently operated in spectrally-dispersea light. By adding wavelength information to high angular resolution, the image of a $\mathrm{Be}$ star can be envisaged as a hypercube of brightness information which depends on the spatial coordinates, wavelength, and eventually polarization and time. A subset of such a representation is shown in Fig. 1.

What OLBI measures is the visibility function $\Gamma e^{i \phi}(u, v, \lambda)$ (right panel), and what we want to determine is the brightness distribution of the source $B(\alpha, \delta, \lambda)$ (left panel). The problem is that the sampling of the right cube is often sparse due to the limited spectral bandwidth and number of interferometric sub-apertures. Therefore, inversion from the measured right cube to the left cube is not straightforward. A natural way to interpret OLBI 
observations is to build the left cube from a physical model, to compute $\Gamma e^{i \phi}(u, v, \lambda)$ from it, and to iterate on the input parameters of $B$ until $\Gamma$ fits the observations. Since OLBI is a newcomer to the field of Be stars, this method of modelling is not familiar to most theoreticians, but hopefully the situation will change in the coming years.

Traditionally, a two-telescope interferometer can measure only the modulus of the Fourier transform of sky brightness and only provides dimensional information concerning the star of interest. If the interference pattern is dispersed by a spectrograph, one can simultaneously observe in as many spectral channels as can be resolved across the total bandwidth. In practice, the only limitation to this technique comes from the detector size and the signal-to-noise ratio. Thus we can compare the size of the program star at different wavelengths and detect chromatic effects. In addition, we can measure the relative phase of the interferometric signal at different Doppler shifts along an emission line compared with the continuum. To first order, the detected phase offsets, if any, correspond to differences of position on the sky. They tell us about the spatial distribution of iso-radial velocity regions in the envelope.

However, these methods should be used with caution. For instance, if the continuum object is partially resolved it does not provide a safe signal for calibrating the visibility across the line. Be-star models (Poeckert \& Marlborough 1978; PM hereafter) estimate a significant contribution of the envelope to continuum flux due to free-free emission and to scattering of stellar photons by the envelope. Nevertheless, the extent of the envelope region producing this emission must lie close to the star. The corresponding visibility contribution of the envelope weakly contaminates the continuum visibility. Therefore differential calibration techniques between the line and continuum apply for the present range of baselines of operational interferometers (Stee \& Araujo 1994).

\section{Expected Angular Diameter of Be Star Envelopes from Spectroscopy}

Operational optical interferometers are unlikely to resolve the photospheric diameter of even the brightest Be stars (Table 1). This becomes evident from a study of stellar angular diameters calculated from colour indices (Ochsenbein \& Halbwachs 1982). Accordingly, all Be stars are smaller than 1 mas. Thus a factor 10 improvement in angular resolution, corresponding to kilometric baselines, is necessary for measuring stars up to the 6th visual magnitude. Though the construction and operation of such an instrument remains a real challenge, it would be highly desirable. For instance, the measurement of the angular diameters of both the Be photosphere and the envelope will provide a check on the effectiveness of spectroscopic methods 
which estimate the extent of the envelope from the separation between the peaks of emission profiles (Huang 1972). This method is widely used for deriving the inner and outer extent of Balmer and Fe II emitting envelopes (Hanuschik et al. 1988, Slettebak et al. 1992) with an uncertainty of about 20 percent (Jaschek \& Jaschek 1993). The interaction between OLBI observations and spectroscopy would refine this method and might provide us with an independent means of inferring the distance of Be stars.

\section{TABLE I}

Expected angular diameters for photospheric and circumstellar envelopes of 8 northern hemisphere Be stars which have so far been observed by means of OLBI. The envelope extends in $\mathrm{H} \alpha$ and $\mathrm{H} \beta$ are given in mas where possible, and otherwise in stellar radii $\left(r_{*}\right)$.

\begin{tabular}{llllll}
\hline Star & Spec.type & $m_{v}$ & $\Phi_{*}$ & $\Phi_{H \alpha}$ & $\Phi_{H \beta}$ \\
\hline & & & & & \\
$\gamma$ Cas & B0.5IV & 2.47 & 0.45 & 8.6 & 4.2 \\
$\phi$ Per & B1V & 4.0 & binary & $23.8\left(r_{*}\right)$ & $13.8\left(r_{*}\right)$ \\
$\psi$ Per & B5III & 4.2 & $?$ & $18.1\left(r_{*}\right)$ & $10.5\left(r_{*}\right)$ \\
$\eta$ Tau & B7III & 2.9 & 0.63 & 12.0 & 2.6 \\
48 Per & B3V & 4.0 & $?$ & $?$ & $34.8\left(r_{*}\right)$ \\
$\zeta$ Tau & B1IV & 3.1 & 0.40 & 6.5 & 5.5 \\
$\beta$ CMi & B8V & 2.9 & 0.63 & 10 & 4.2 \\
o And & B6III & 3.6 & quadruple & $1.4\left(r_{*}\right)$ & $0.9\left(r_{*}\right)$ \\
\hline
\end{tabular}

\section{Measured Angular Diameters of Be-Star Envelopes by OLBI}

\section{1. $\gamma$ CAS}

Attempts to measure Be stars can be traced back to the early 1980 s with the observations of the I2T. Using a maximum baseline of $48 \mathrm{~m}$ and a visual method with 10-20 percent uncertainties, the I2T was able to set an upper limit of 0.9 mas on the continuum angular diameter of $\gamma$ Cas (Vakili et al. 1984). In 1985, photoelectric observations of $\gamma$ Cas which combined spatial and spectral resolutions were able to resolve the $\mathrm{H} \alpha$ emitting envelope with intermediate baselines of the order of $20 \mathrm{~m}$ (Thom et al. 1986). The use of the relative visibility phase between the red and blue wings of $\mathrm{H} \alpha$ enabled the I2T to find that the receding regions of the envelope were to the north of the star and the approaching regions were to the south. Furthermore, the I2 $\mathrm{T}$ found that the envelope asymmetry must be smaller than 0.7 stellar radii at the time of observations. 

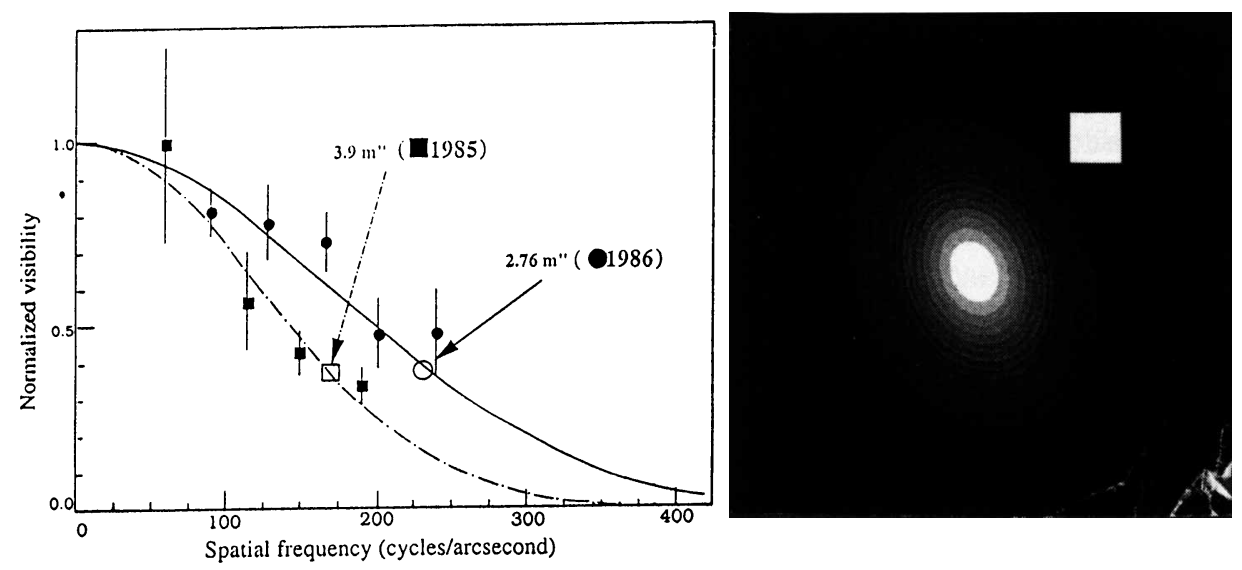

Fig. 2. Results from OLBI observations of $\gamma$ Cas. Left: the angular diameter of the $\mathrm{H} \alpha$ emitting envelope measured by I2T. The size of the envelope, assumed to have a gaussian limb-darkening law, was found to be about eight times larger than the photosphere and most probably shrank in size within a one year interval from 1985-1986. Right: a major contribution by Mark III observations to the understanding of $\gamma$ Cas using earth-rotation synthesis. The angular scale is given by the white square on the top right which corresponds to 1 mas. North is to the top, east to the left. This $\mathrm{H} \alpha$ image was computed by the author using recent results published by Quirrenbach et al. (1993).

There is a long-standing controversy concerning the mechanism which produces Balmer line $\mathrm{V} / \mathrm{R}$ variations. Such observations, which are of particular interest, were conducted on $\gamma$ Cas in 1986. They indicated that the envelope had shrunk by about 10 percent since 1985 (Percheron et al. 1994), while $\mathrm{V} / \mathrm{R}$ in $\mathrm{H} \alpha$ increased during the same period (Doazan et al. 1987). If confirmed, this isolated observation (Fig. 2) is more consistent with the pulsating envelope scenario than with the more popular precessing ellipticaldisk model (Tetling et. al. 1993). Unpublished observations of $\gamma$ Cas in 1986 have also set an upper limit of 4 mas to the envelope in the $\mathrm{H} \beta$ line (Vakili 1990).

More recent observations of $\gamma$ Cas by the GI2T (Mourard et al. 1989) not only confirmed the 1985 results of the I2T, but opened new perspectives for studying the kinematics of Be-star envelopes. With an improved spectral resolution of as much as $0.2 \mathrm{~nm}$, and with twice the angular resolution of the I2T, Mourard and co-workers discovered morphological structures of the envelope through the study of the modulus and phase variations of the visibility across $\mathrm{H} \alpha$. As many as 7 contiguous spectral channels were obtained across the $\mathrm{H} \alpha$ line (Fig. 3). The interpretation of these observations were based on the PM model of $\gamma$ Cas. The objection could be made that at the 

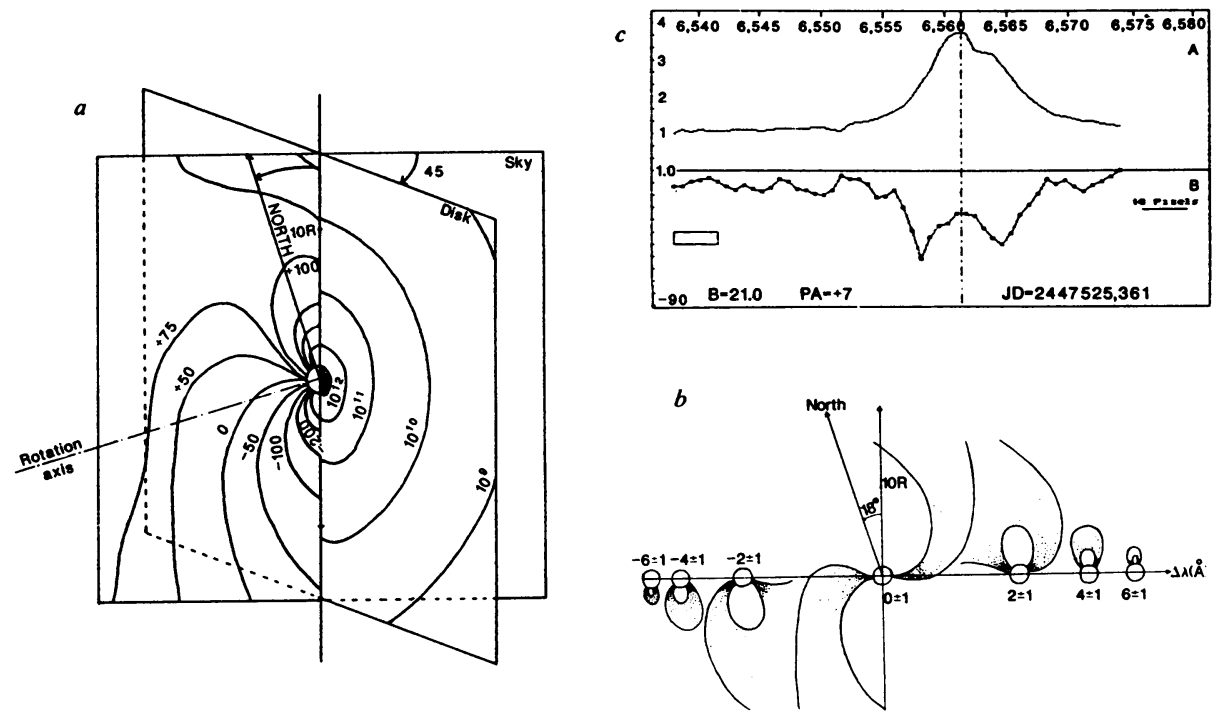

Fig. 3. Interpretation of spectrally resolved OLBI observations of $\gamma$ Cas according to Mourard et al. (1989). a) Iso-radial velocity contours in the sky plane obtained from the projection of the velocity law computed by Poeckert \& Marlborough (1978). b) Inferred intensity maps corresponding to different iso-radial velocity areas of the envelope. c) Observed $\mathrm{H} \alpha$ profile (upper curve) and corresponding visibilities as a function of doppler-shift across the line (below).

time of the observations the emission profile of $\mathrm{H} \alpha$ was in a $\mathrm{V}>\mathrm{R}$ regime, so that the PM model could not apply. However, this model was used merely as a guideline and the conclusion was that the measurements agreed well with a rotating envelope. The GI2T results showed that it is not easy to compare OLBI observations with any presently existing Be model. Unless model builders modify their code to produce emission line intensity maps from which visibilities can be predicted, OLBI observations have no straightforward interpretation. The situation will be different once sub-milliarcsecond maps are derived directly from multi-aperture OLBI.

A major step towards this target has been taken by the Mark III interferometer. Using earth-rotation synthesis exceeding $100^{\circ}$ for some baselines, Quirrenbach et al. (1993) have clearly shown that the envelope of $\gamma$ Cas is oblate. Their observations, made through a $1 \mathrm{~nm} \mathrm{H} \alpha$ filter, were fitted to an elliptical Gaussian with an axial ratio of 0.74 and a FWHM major axis diameter of 3.2 mas (Fig. 2). This agrees well with the I2T and GI2T results and, besides establishing the disklike geometry of the envelope, also provides 


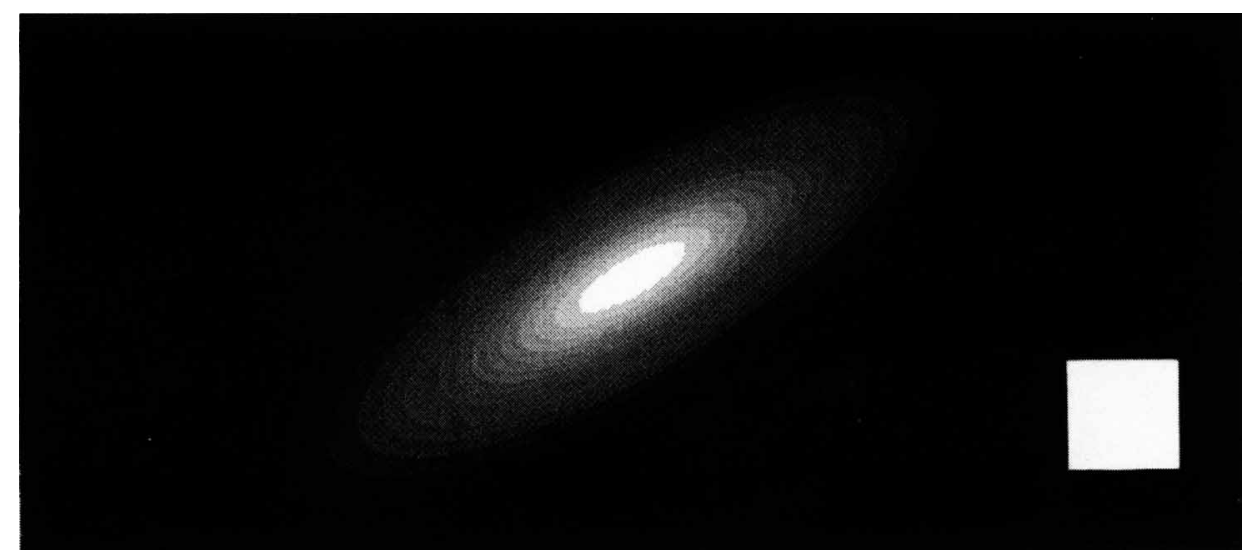

Fig. 4. The H $\alpha$ map of $\zeta$ Tau modelled as an elliptical Gaussian. The major axis is tilted by $59^{\circ} \mathrm{NW}$. North is at the top and east to the left. The white square gives the angular scale of the map and corresponds to 1.2 mas on the sky.

the orientation of the rotational axis. The $21^{\circ} \mathrm{NE}$ tilt is in excellent agreement with the $20^{\circ}$ positional angle obtained by polarimetry. The oblateness of $\gamma$ Cas is indisputable: even raw visibilities bear a clear signature of the asymmetry (see Fig. 2 of Quirrenbach et al. 1993). However, the question of asymmetry with respect to the rotation axis remains open until continuous observations, which include the phase information, can be obtained.

\section{2. $\zeta \mathrm{TAU}$}

The first resolution of the Be shell star $\zeta$ Tau is another illustration of OLBI findings with the Mark III interferometer (Quirrenbach et al. 1994). From a Maximum Entropy map reconstructed in $\mathrm{H} \alpha$, Quirrenbach and co-workers deduce a maximum elongation of $(3.55 \pm 0.33)$ mas, tilted by $59^{\circ} \mathrm{NW}$ (Fig. 4). By considering the perspective, they also determine the inclination angle of the rotational axis and estimate the rotation velocity at $280 \mathrm{~km}$ $\mathrm{s}^{-1}$, which is 85 percent of the breakup limit.

\section{Other Results}

Besides the resolution of $\gamma$ Cas and $\zeta$ Tau in $\mathrm{H} \alpha$, observations of 5 other $\mathrm{Be}$ stars by Mark III are reported by Quirrenbach (these Proceedings). These results confirm the asymmetry of two other objects, $\psi$ Per and $\phi$ Per, and indicate that 48 Per and $\eta$ Tau are seen almost pole on. It also appears that envelope oblateness is clearly correlated with $v \sin i$ and that the orientations of the major axes are in excellent agreement with polarimetric observations. This supports the hypothesis of electron scattering in a disklike envelope. 


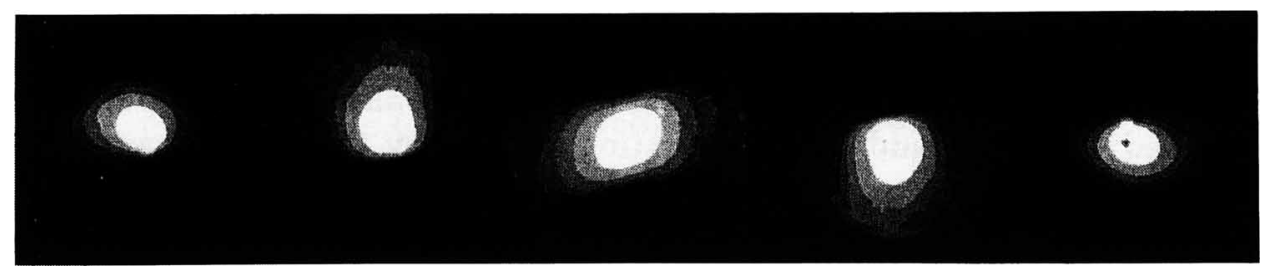

Fig. 5. Intensity maps at various Doppler-shifts across the $\mathrm{H} \alpha$ emission line for a $\mathrm{B} 1 \mathrm{Ve}$ star and computed from a rotating expanding-envelope model. From left to right: envelope maps computed from $\delta \mathrm{v}=-1000 \mathrm{~km} \mathrm{~s}^{-1}$ to $\delta \mathrm{v}=+1000 \mathrm{~km} \mathrm{~s}^{-1}$ in steps of $400 \mathrm{~km} \mathrm{~s}^{-1}$ and averaged on spectral channels each $500 \mathrm{~km} \mathrm{~s}^{-1}$ wide. The rotation is $45^{\circ}$ along a vertical axis. Such maps predicted by Be-models are highly desirable for direct interpretation of OLBI observations (Courtesy of Stee \& Araujo).

Nevertheless, we must bear in mind that Mark III observations are based on visibility modulus information only: they cannot detect departures from axisymmetry.

Mourard et al. (1992) reported spectrally resolved OLBI observations of $\beta$ Lyr at various orbital phases. Their data include the recording of the $\mathrm{H} \alpha$, $\mathrm{H} \beta$ as well as the $\mathrm{He} \mathrm{I} \lambda 6678$ emission lines obtained with interferometric baselines ranging from $16-51 \mathrm{~m}$. There is no clear indication that the binary system is resolved. There is, however, a partial resolution of the $\mathrm{H} \alpha$ region which is centered on the continuum object, whilst the He I region remains unresolved.

Time-resolved spectroscopy of Pleione during a lunar occultation has been reported by Gies et al. (1990), who detect a time lag between the occultation of the continuum and the emission line. This is interpreted as an asymmetry of the envelope extending to $34-55$ stellar radii which would be formed by gravitational influence of the binary companion discovered recently by speckle-interferometry.

\section{Targets for the Future}

\subsection{The Temporal Variation of Be Stars}

The temporal variability of Be stars has different time scales and amplitudes. On one extreme, variations of a few minutes across the $\mathrm{H} \alpha$ profiles have been reported by Anandarao (these Proceedings). If these variations result from photospheric activities which propagate through the envelope, local structures of the latter should be modified and presumably become detectable by means of OLBI. However, the characteristic cycles are very short and the interferometric signal can only be integrated over a few minutes. Most 
probably such observations can only be achieved with synthesis arrays of the GI2T class which use sub-apertures of the 1-2 m class. On the other extreme, variations of a few years in $V / R$ are generally attributed to onearmed density waves propagating in nearly Keplerian disks (Okazaki, these Proceedings) as opposed to pulsating envelopes (Doazan 1987). Spectrally resolved OLBI can address this question directly by tracing the variations of the envelope size and correlating it with $V / R$.

\subsection{Polarization and Chromatic Effects}

OLBI also allows high spatial resolution observations in polarized light (Vakili 1981). Simultaneous observations in two different polarizations permit the detection of magnetic structures and phenomena such as electron scattering in the envelope.

Observations with high spectral resolution over a large bandwidth can be used to probe the circumstellar structure. Observing in two bands centered on $\mathrm{H} \alpha$ and $\mathrm{H} \beta$ enables us to disentangle the flux contribution of the star, the free-free emission of the envelope, and the photospheric flux scattered by the envelope. Other phenomena such as starspots or differential rotation could potentially be detected with spectrally resolved OLBI. However, these observations demand an order of magnitude improvement in angular resolution and sensitivity.

\subsection{DiRECT DETECTION OF NRP}

The direct detection of nonradial pulsations (NRP) can be envisaged using two different methods. Using differential speckle or long baseline interferometry we can locate the two-dimensional spatial distribution of iso-radial velocity regions on the stellar disk. This would allow the detection of tesseral as well as sectorial modes (Vakili \& Percheron 1991). Rough estimates of signal to noise ratio (Petrov, private communication), indicate that high degree modes, say $l=-m=12$, are observable with interferometers using 2 -m class telescopes. Another approach consists of placing the array of telescopes to suit the spatial frequency of the pulsations that we wish to detect. As argued by Balona (1990), NRP must modulate at some level the photospheric disk. The spatial filter which is the interferometer can be tuned to the scales of the NRP which we want to detect. The presence of a visibility signal would signify the detection of an NRP mode. Although such observations are not forseeable in the immediate future, it is important to bear them in mind.

\subsection{Towards Interferometric Modelling of Be-star envelopes}

The pioneering work at OCA and the achievements of Mark III clearly demonstrate that direct constraints provided by OLBI are applicable. Several optical aperture synthesis arrays will start operating in the coming decade (Robertson \& Tango 1994). Many ad hoc or physical models have 
been reported during this symposium. Theoreticians take note: as with photometry, polarimetry, and spectrometry, models for Be-star envelopes should provide observable parameters for interferometry!

\section{Acknowledgements}

The authors are indebted to $P$. Lawson for his careful reading of the manuscript and to L. Balona for his generous help during the editing. We acknowledge A. Quirrenbach and D. Bonneau for having kindly provided us with data from Mark III and speckle-interferometric observations.

\section{References}

Balona, L.: 1990, Mon. Not. Roy. Astr. Soc. 245, 92.

Blazit A., Bonneau, D., Koechlin, L. and Labeyrie, A.: 1977, Astrophys. J. Let. 214, 79.

Bonneau, D., Blazit D., Foy, R. and Labeyrie, A.: 1980, Astron. Astrophys. Suppl. $42,185$.

Cassinelli, J. and Hoffman, J.P.: 1975, Mon. Not. Roy. Astr. Soc. 173, 789.

Doazan, V., Rusconi, L., Sedmak, G., Thomas, R.N. and Bourdonneau, B.: 1987, Astron. Astrophys. 182, L25.

Doazan, V.: 1987, in Slettebak, A. and Snow, T.P., eds., IAU Colloq. 92: Physics of Be stars, Cambridge Univ. Press: Cambridge, 384.

Gies, D.R., McKibben, W.P., Kelton, P.W., Opal, C.B. and Sawyer. S.: 1990, Publ. Astr. Soc. Pacific 100, 1601.

Hanbury Brown, R., Davis, J., Herbison-Evans D. and Allen, L.R.: 1970, Mon. Not. Roy. Astr. Soc. 148, 103.

Hanbury Brown, R., Davis, J. and Allen, L.R.: 1974a, Mon. Not. Roy. Astr. Soc. 167, 121.

Hanbury Brown, R., Davis, J. and Allen, L.R.: 1974b, Mon. Not. Roy. Astr. Soc. 168, 93.

Hanuschik, R.W., Kozok, J.R. and Kaiser, D.: 1988, Astron. Astrophys. 189, 147

Huang S.S: 1972, Astrophys. J. 171, 549

Jaschek, C and Jaschek, M.: 1993, Astron. Astrophys. Suppl. 97, 807.

Labeyrie, A.: 1978, Ann. Rev. Astron. Astrophys. 16, 77.

Labeyrie, A., Bonneau, D., Stachnik, R.V. and Gezari. D.Y.: 1974, Astrophys. J. Let. 214, 79.

McAlister, H. and Hartkopf, W.I.: 1988, Second Catalog of Interferometric Measurements of Binary Stars, CHARA Contribution No. 2.

Mourard, D., Bosc, I., Labeyrie, A., Koechlin, L. and Saha, S.: 1989, Nature 342, 520.

Mourard, D., Bonneau, D., Blazit, A., Labeyrie, A., Morand, F., Percheron, I., TallonBosc, I., Vakili, F.: 1992, in McAlister, H.A. \& Hartkopf, W.I., eds., IAU Colloq. 135, Complementary Approaches to Double and Multiple Star Research, ASP Conf. Series, 32

Ochsenbein, F and Halbwachs, J.L.: 1982, Astron. Astrophys. Suppl. 47, 523.

Percheron, I., Rabbia, Y. and Vakili, F.: 1994, Astron. Astrophys. submitted.

Poeckert, R. and Marlborough, J.M.: 1976, Astrophys. J. 206, 182.

Poeckert, R. and Marlborough, J.M.: 1978, Astrophys. J. 220, 940 (PM).

Quirrenbach, A., Hummel., C.A., Buscher, D.F., Armstrong, J.T., Mazurkewich, D. and Elias, N.M.: 1993, Astrophys. J. Let. 416, 25.

Quirrenbach, A., Buscher, D.F., Mazurkewich, D., Hummel, C.A. and Armstrong, J.T.: 1994, Astron. Astrophys. submitted.

Robertson J.G. and Tango, W.J.: 1994, IAU Colloq. 158, Very High Angular Resolution Imaging, Kluwer: Dordrecht.

Slettebak, A., Collins, G.W. and Truax, R.: 1992, Astrophys. J. Suppl. 81, 335. 
Stee, P. and Araujo, X.F.: 1994, Astron. Astrophys. submitted.

Tetling T.J.H., Waters L.B.F.M., Persi P. and Dunlop S.R.: 1993, Astron. Astrophys. 270, 355.

Thom, C., Granes, P. and Vakili, F.: 1986, Astron. Astrophys. 165, L13.

Vakili, F.: 1981, Astron. Astrophys. 101, 352.

Vakili, F.: 1990, PhD. Thesis, University of Nice

Vakili, F., Granes, P., Bonneau, D., Noguchi, M. and Hirata, R.: 1984, Publ. Astr. Soc. Japan 36, 231.

Vakili, F. and Percheron, I.: 1991, in Baade, D., ed., Rapid Variability of OB-Stars: Nature and Diagnostic Value, ESO: Garching, 77.

\section{Discussion}

Gies: In $\phi$. Per has the binary companion been resolved?

Quirrenbach: The Mark III observations have not seen the signature of the companion in the continuum data and we concluded that probably $\Delta \mathrm{m}$ $\geq 2.5$.

Vakili: $\phi$ Per's companion was once resolved by speckle-interferometric observations on the Palomar 5-m telescope in 1975. On JD=2442643.5 Bonneau (private communication) estimated a separation of $\rho=20$ mas, $\theta=$ $330^{\circ}$ and $2<\Delta \mathrm{m}<3$.

Smith: We are looking forward to the day when you can resolve the inner radius of the disk to see whether it is distinct from the stellar disk.

Vakili: Even the brightest Be stars are smaller than 1 mas and will require interferometric baselines exceeding a few hundred meters. One might address these questions by using Differential Speckle Interferometry introduced by J. Beckers. In principle DSI attains sub-milliarcsecond resolutions even with monolithic telescopes of the 10-m class.

Sareyan: Is it true that with existing techniques any asymmetry in the $\mathrm{H} \alpha$ envelope would appear as symmetrical in the image reconstruction?

Quirrenbach: Reconstructed images from Mark III observations are based on visibility modulus data from earth-rotation synthesis. To get a final answer on asymmetries, phase data are clearly needed.

Friedjung: Do stars with elliptical images tend to have shell episodes and do they tend to have a higher $v \sin i$ than those with circular images?

Vakili: Ellipicities determined for 6 bright northern Be stars by Quirrenbach and co-workers correlate with the degree of polarization that one would expect from equatorially-flattened disks. The correlation between the degree of polarization and $v \sin i$ is now well established (e.g., Poeckert \& Marlborough 1976). Shell features are not permanent in Be stars and interferometric observations should be repeated as often as possible to track any variation in the shape of the envelope. 
Hirata: Can spectrally-resolved interferometry provide any information on the circular velocity field, i.e., Keplerian or otherwise?

Vakili: Mourard et al. (1989) have actually done this on $\gamma$ Cas some 4 years ago with the GI2T. Other interferometers under construction such as the Big Optical Array will also observe in dispersed light. In order to obtain the exact dynamics of the envelope, models should provide the intensity map of iso-radial velocity areas at a given Doppler shift along the emission profile. Predicted visibilities could thus be directly compared to interferometric measurements and vice-versa.

Harmanec: Would an unrecognized radial velocity of say, the $\mathrm{H} \alpha$ line, fool your image reconstruction?

Quirrenbach: In addition to the $1 \mathrm{~nm}$-wide filter, we have also used a 10 $\mathrm{nm}$-wide filter centered on $\mathrm{H} \alpha$. The results of the two filters agree with each other in all cases.

Vakili: If the $\mathrm{H} \alpha$ extends beyond the spectral width of the narrow filter, the corresponding iso-radial velocity areas are rejected and the reconstructed image is incomplete. This is obviously true for Be stars with large projected rotational velocities, especially when the emission is strongly asymmetric as in $\zeta$ Tau.

Arsenijevic: Could you please make some comments on the necessity to have additional information, i.e. to use different techniques simultaneously (photometry, spectroscopy, polarimetry, etc.)?

Vakili: In principle, all these techniques can be combined on an optical interferometer by observing in polarized and spectrally dispersed light. Nevertheless, simultaneous observations by more well-developed techniques are required for calibrating the data from long baseline interferometry. Most of all, they can monitor the observational strategies of interferometry, for instance when active episodes occur on target Be stars. 